\title{
The Drosophila female-specific sex- determination gene, Sex-lethal, has stage-, tissue-, and sex-specific RNAs suggesting multiple modes of regulation
}

\author{
Helen K. Salz, ${ }^{1,2}$ Eleanor M. Maine, ${ }^{1,3}$ Linda N. Keyes, ${ }^{1}$ Mark E. Samuels, ${ }^{1}$ Thomas W. Cline, ${ }^{1}$ and \\ Paul Schedl ${ }^{1}$ \\ ${ }^{1}$ Department of Biology, Princeton University, Princeton, New Jersey 08544 USA; ${ }^{2}$ Department of Genetics, Case Western \\ Reserve University, School of Medicine, Cleveland, Ohio 44106 USA
}

\begin{abstract}
For proper sexual development of females, the Sex-lethal (Sxl) gene must be activated early in development and remain on during the rest of the life cycle. Conversely, in males, $S x l$ must remain functionally off through development. Here, we show that the Sxl transcription unit spans a DNA segment of $>20 \mathrm{~kb}$ and encodes at least 10 distinct, but overlapping, RNA species. These RNAs range in size from 4.4 to $1.7 \mathrm{~kb}$ and exhibit sex, stage, and tissue specificity. Six RNAs, three female specific and three male specific, are first detected by midembryogensis and persist through the adult stage: Their expression reflects the on/off regulation of Sxl's activity at the level of sex-specific alternate splicing. Four Sxl RNAs are found in adult females. Two of these RNAs are dependent on the presence of a functional germ line and may be relevant to $S x l$ 's role in adult germ-line development. All four are present in unfertilized eggs. Finally, three Sxl RNAs are found only transiently during very early embryogenesis; we suggest that the expression of these RNAs may reflect an early regulation of $S x l$ at the level of transcription and that these transcripts are involved in the initial selection of the $S x l$ activity state in response to the primary sex-determination signal, the $\mathrm{X} / \mathrm{A}$ ratio.
\end{abstract}

[Key Words: Drosophila melanogaster; Sex-lethal; sex determination; dosage compensation; sex-specific transcripts]

Received January 24, 1989; revised version accepted March 1, 1989.

The binary switch gene Sex-lethal (Sxl) plays a central role in the development of sexual dimorphism in the fruit fly Drosophila melanogaster (for review, see Baker and Belote 1983; Cline 1985, 1988b; Nöthiger and Steinmann-Zwicky 1985). The activity state of $S x l$ determines which developmental pathway is followed: The 'on' state imposes female development, whereas the 'off' state allows male development. Uniquely among all the genes known to be involved in controlling sexual dimorphism in fruit flies, $S x l$ participates in three different levels of developmental regulation: the initial selection of the developmental pathway (initiation), the maintenance of a heritable commitment to the pathway (determination) and, finally, the expression of the pathway (differentiation).

Much of our current understanding of how $S x l$ functions in these different developmental processes comes from analysis of the phenotypes and complementation patterns of various mutant alleles and from genetic studies on Sxl's interactions with both upstream and

${ }^{3}$ Present address: Laboratory of Molecular Biology, University of Wisconsin, Madison, Wisconsin 53706 USA. downstream genes in the pathway. As with other complex loci of Drosophila, mutant alleles of Sxl exhibit a complicated pattern of complementation. This complementation pattern is most easily understood as reflecting a diversity of functional $S x l$ products on the one hand and distinct modes of $S x l$ regulation during the life cycle on the other hand. In this paper we begin to explore the molecular correlates of this complexity.

A variety of lines of indirect evidence suggest that the initial selection of the female or male $S x l$ activity state occurs early in embryogenesis in response to the number of $\mathrm{X}$ chromosomes relative to the number of sets of autosomes (X/A ratio) (Sanchez and Nöthiger, 1983; Cline 1984; Gergen 1987). An X/A ratio of one (XX) signals the activation of the $S x l$ gene, whereas it is not activated when the X/A ratio is one-half [(XY); see Cline 1988a]. The activation of $S x l$ depends on factors in addition to the $\mathrm{X} / \mathrm{A}$ ratio such as the daughterless gene product (Cline 1983; Gergen 1987). Another trans-acting factor involved in early $S x l$ regulation may be generated from the zygotic $S x l$ gene. Analysis of various $S x l$ alleles suggests that this gene has early embryonic functions that are genetically separable from the functions of the gene 
later in development. One of these appears to be required for the stable activation of the Sxl gene (Cline 1984, 1988a,b; Maine et al. 1985b; Salz et al. 1987). Another very early function appears to be involved in the down-regulation of the $\mathrm{X}$ chromosome dosage-compensation system (see Gergen 1987).

Once the initiation process is complete and $S x l$ is on in the female embryo, the maintenance of a commitment to the female pathway ('determination' in the strict developmental sense) requires that $S x l$ remain in an active state from embryogenesis through the adult stages. The stable commitment to the female pathway is thought to be achieved via a positive autoregulatory function of Sxl, which keeps it in an active mode (Cline 1984, 1988a,b; Maine et al. 1985b; Bell et al. 1988). The other level is 'differentiation.' $\mathrm{Sxl}$ is required at later stages in development for the proper expression of the female developmental pathway (Cline 1979a, 1984; Sanchez and Nöthiger 1982). Genetic and molecular studies have shown that $S x l$ controls the activity state of genes downstream in the sex-determination pathway and dosage compensation system. The most immediate known target of $S x l$ in the sex-determination pathway is transformer (tra) (Cline 1979b; Baker and Ridge 1980; Nagoshi et al. 1988). Like Sxl, tra must be on in females but off in males. The on/off regulation of tra is posttranscriptional and involves the sex-specific splicing of the tra RNA (Boggs et al. 1987). Moreover, it has been suggested that Sxl may turn on tra by directing the female-specific splicing of the tra transcript (Boggs et al. 1987; Bell et al. 1988; Nagoshi et al. 1988). Sxl also is required to keep the dosage-compensation system off in females (Lucchesi and Skripsky 1981; Cline 1983, 1984; Gergen 1987). Although a number of genes involved in regulating some aspects of dosage compensation have been characterized (for review, see Lucchesi and Manning 1987), the most immediate target(s) for Sxl's action is not known (Cline 1984; Gergen 1987). In addition to these somatic functions, $S x l$ is also required for the normal development of the female germ line (Schüpbach 1985; Salz et al. 1987).

To understand more fully the role of $S x l$ in the development of sexual dimorphism, we have undertaken a molecular analysis of the gene. The $S_{x l}$ gene was isolated initially using P-element tagging and chromosome walking (Maine et al. 1985a). In the work reported here, we have extended the initial chromosomal walk and have examined the transcripts encoded by a $\sim 44-\mathrm{kb}$ DNA segment around the $S \times l$ locus in both wild-type and mutant $S x l$ alleles. These studies have permitted the identification of the $S x l$ transcription unit and indicate that it covers a DNA segment of $\sim 20 \mathrm{~kb}$. (This analysis substantially revises the preliminary map of the transcription unit reported in Maine et al. 1985b). We find that the functional complexity of $S x l$ evident in genetic studies is paralleled at the molecular level: $S x l$ encodes a complex set of transcripts that show sex, stage, and tissue specificity. The analysis of RNAs in female and male animals reported here provided the first evidence for the sex-specific processing of $S x l$ transcripts and sug- gests that it occurs throughout most of the life cycle. Studies on Sxl cDNAs from adult animals reported elsewhere (Bell et al. 1988) have confirmed the sex-specific processing of the $S x l$ transcripts and indicate that alternate splicing is a mechanism for the on/off regulation of the gene. The complex transcription pattern also affords the first hints as to the molecular basis for the sex-specific activation of $S x l$ very early in development and its possible relationship to the regulation and function of the gene later in development.

\section{Results}

\section{Localization of the Sxl transcription unit}

A physical map of the $\sim 44-\mathrm{kb}$ DNA segment from the cytogenetic region $6 \mathrm{~F}$, which contains the $S x l$ gene, is shown in Figure 1. In our initial characterization of this DNA segment (Maine et al. 1985a), we found that DNA rearrangements associated with $S x l$ mutations were clustered in an 11-kb DNA segment extending from coordinate 0 to -11 . We further defined sequences required for the various $S x l$ functions by analyzing a series of deficiencies in the $6 \mathrm{~F}$ region generated by the mobilization of a P element $\left(S x f^{f P b}\right)$ inserted at coordinate 0 (Salz et al. 1987). The limits of some of these deficiencies within the chromosomal walk are indicated at the top of Figure 1.

To identify transcribed sequences in the 6F DNA segment, we used nick-translated restriction fragments to probe Northern blots of poly $(\mathrm{A})^{+}$RNA prepared from 0 to 5 -hr embryos and adult females and males. The results of this analysis are summarized at the bottom of Figure 1. At least 16 different RNA species are detected with probes from the $44-\mathrm{kb}$ region. Because $S x 1$ is required in females but not in males and also must be active in very young embryos, a comparison of the transcripts hybridizing to the various probes in the two sexes and at the different stages should suggest which of these transcripts might be products of the $S \times l$ gene. On the basis of these criteria, the transcripts encoded by sequences on the proximal and distal sides of our walk would not appear to be good candidates for $S_{x} 1$ RNAs. On the centromere proximal side (left), e.g., probes a-d (extending from coordinates +15 to +0.5 ) hybridize to four different RNA species that are present in adults of both sexes and are also found in early embryos. On the distal side (right) probe 1 labels two poly(A) ${ }^{+}$RNAs. These are also found in adult males and females but are absent in early embryos.

A different result is obtained with probes derived from near the middle of the walk. Fragments from a region extending from coordinates 0 to -22 hybridize to a complex array of overlapping transcripts that show both sex and stage specificity. A blot probed with fragment $h$ (see Fig. 1), which hybridizes to all of the major transcripts from this region, is shown in Figure 2. In adult females, three major size classes of RNA are observed: $4.2,3.3$, and $1.9 \mathrm{~kb}$. A similar set of three RNA size classes is found in adult males; however, as can be seen 


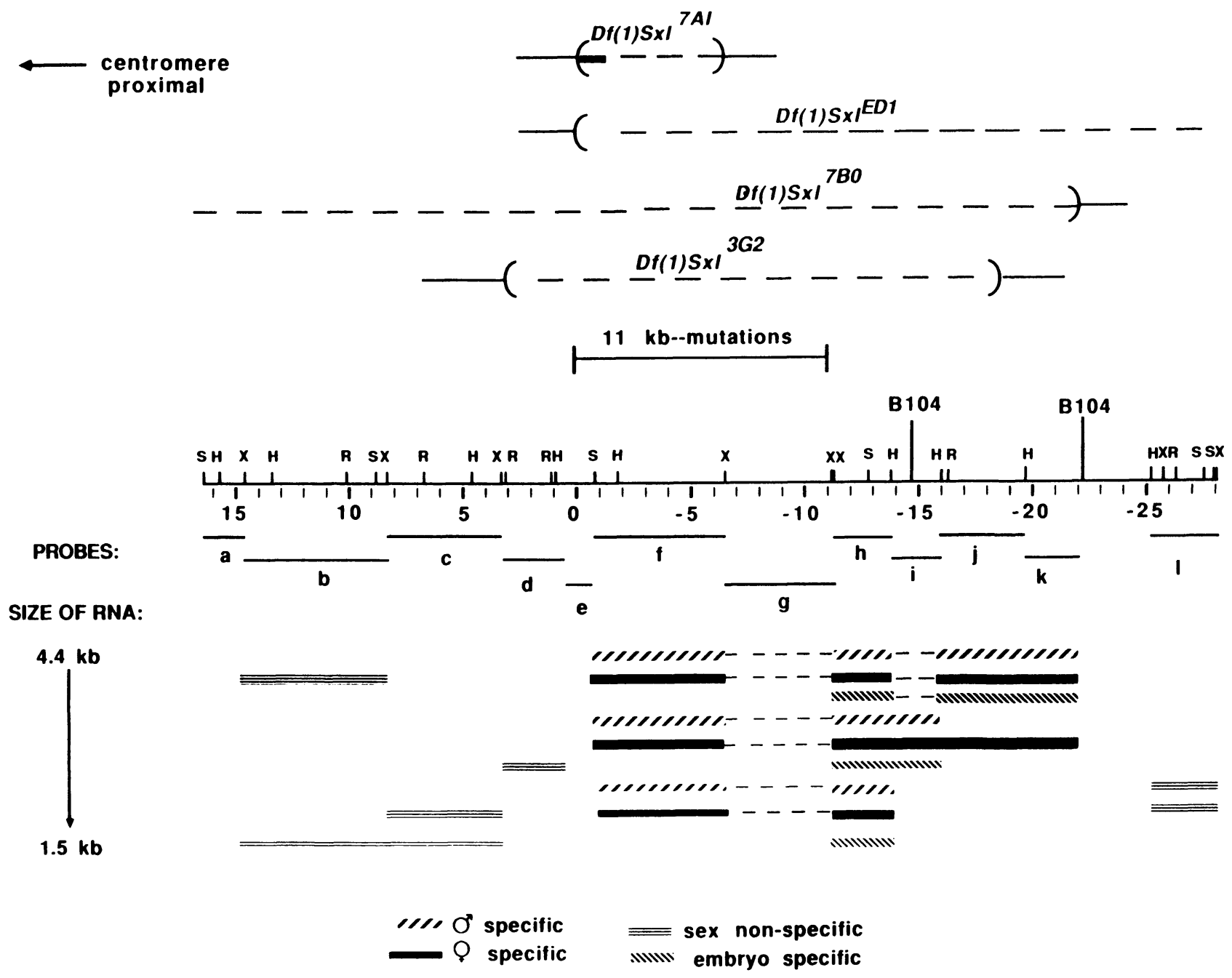

Figure 1. Restriction map, transcribed regions, and chromosome rearrangements within the $\sim 44-\mathrm{kb}$ segment that includes $S \times 1$. The fragments used as probes in this analysis are labeled a-l. The transcripts detected by each fragment are indicated by the solid bars directly below the fragment in decreasing order of size (the actual region of hybridization may be only a small portion of that fragment, e.g., see Fig. 4). Those transcripts of the same size and temporal and sex specificity are presumed to correspond to a single RNA species and are joined by a dashed line to show the putative introns. Although this analysis only detected 15 transcripts within this region, subsequent analysis (see Figs. 4 and 7) demonstrated that there are 16 RNAs, 2 of which comigrate at $3.3 \mathrm{~kb}$. The location of the 11-kb region that contains DNA rearrangements associated with $S x l$ mutations previously characterized by Maine et al. (1985a) is shown. Also shown are the breakpoints of several Sxl deletion chromosomes used in this study. The dashed lines in the deletion chromosomes indicate the limits of the deletion as determined by genomic Southern blots (Salz et al. 1987 and P. Schedl, unpubl.). The thick solid rectangle in $D f(1) S x l^{7 A 1}$ is a P element. The two B104/roo elements (Meyerowitz and Hogness 1982; Scherer et al. 1982) shown on the map are not associated with a mutant phenotype. Some wild-type strains have both of these elements, some have only one and some have neither insertion. Note that we reversed the orientation of the restriction map relative to that described in Maine et al. (1985a,b) and Salz et al. (1987) so that the 5' end of Sxl is on the left. Restriction sites are designated as follows: (R) EcoRI; (X) XhoI; (H) HindIII; (S) SalI.

in Figure 2, the male-specific RNA species are 100-300 bp larger than the corresponding female RNAs $(4.4,3.6$, and $2.0 \mathrm{~kb})$ in each case. The transcript pattern in 0 - to 5 -hr embryos is more complex. We observe three RNAs which, by mobility, appear to correspond to the three major size classes of RNA found in adult females. In addition, there are two bands of 3.1 and $1.7 \mathrm{~kb}$, respectively, which are only observed in embryos. On higher percentage gels, a third embryo-specific transcript of $\sim 4.0 \mathrm{~kb}$ can be resolved from the large 4.2 $\mathrm{kb}$ female-specific transcript (data not shown).
Several lines of evidence indicate that this complex group of overlapping RNAs corresponds to the $S \times 1$ transcripts. First, the transcription unit that would encode these RNAs includes the $11-\mathrm{kb}$ region (see Fig. 1) that contains the $S x l$ DNA rearrangements used in the initial identification of the locus (Maine et al. 1985a). Second, we have found that a number of these loss-of-function mutations alter the $S x l$ transcript pattern. (Because these mutations are female lethal, the $S x l$ transcripts were examined in adult males.) For example, all three male-specific transcripts are absent in adult males carrying the 


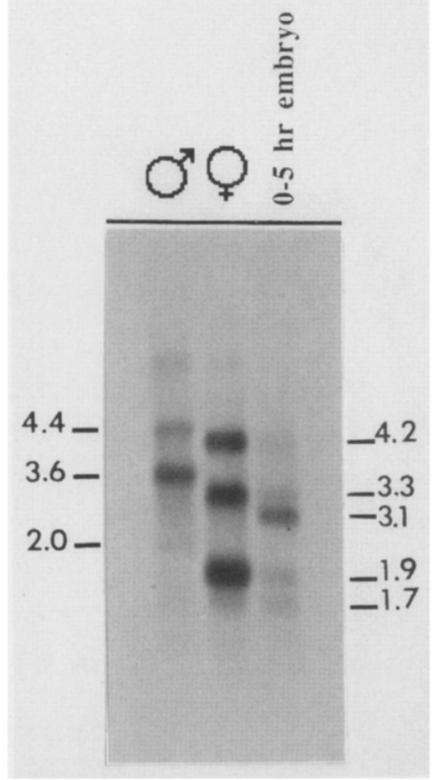

Figure 2. Sxl transcripts in adult females, adult males, and 0to 5-hour embryos. The Northern blot was hybridized with fragment $\mathrm{h}$ (Fig. 1). Poly(A) ${ }^{+}$adult RNA $(5 \mu \mathrm{g})$ or poly(A) ${ }^{+}$embryo RNA $(2.5 \mu \mathrm{g})$ was loaded per lane. Occasionally we observe a larger $(\sim 7 \mathrm{~kb}) \mathrm{RNA}$, which may be an incompletely processed RNA.

loss-of-function mutation $S x{ }^{f P b}$ (which has a P-element transposon inserted at coordinate 0 ). In contrast, the sex nonspecific RNA species encoded by sequences just proximal to coordinate 0 (e.g., the 2-kb RNA of fragment $\mathrm{d}$ or the 4-kb RNA of fragment b: see Fig. 1) are unaffected by the P-element insertion (data not shown).

This conclusion is supported by analysis of the transcripts produced by males carrying small deletions that affect $S x l$ function. In the experiment presented in Figure 3, RNA was prepared from four Sxl deficiency mutations and probed with either fragment $\mathrm{h} 2$ [which hybridizes strongly to the small and middle RNA species (2- and 3-kb transcripts) but only weakly to the large (4-kb) RNAs (see Fig. 4)] or with fragment d (which labels the proximal 2.0-kb sex-nonspecific transcript). As indicated in Figure 1, two of these deficiencies, $7 B O$ and $3 G 2$, completely lack both fragment $h$ and d DNA. As would be expected, the three male-specific Sxl transcripts and the 2.0-kb RNA are missing in these two deletion mutants (see Fig. 3). In other experiments (data not shown) we have found that the more proximal 4.2-, $1.9-$, and 1.5-kb RNAs (see Fig. 1) are still present in males carrying the smaller deletion $3 G 2$.

Deficiency $E D 1$ has fragment d sequences but is deleted for fragment h2, as well as more distal sequences beyond the endpoint of our chromosomal walk (Fig. 1). As can be seen in Figure 3, the 2.0-kb proximal RNA is expressed in males carrying this deficiency, whereas none of the male $S x l$ RNAs is observed. The smallest deletion, $S x l^{7 A 1}$, lacks sequences extending from coordinate 0 to -6.5 but retains fragment h DNA. Adult males carrying this small deficiency express the proximal sex nonspecific 2-kb RNA (data not shown). In contrast, the male-specific transcripts are absent in RNA from this mutant (Fig. 3b), and instead we observe a weakly labeled smear of hybridization (which is more evident in longer exposures). Because the proximal breakpoint of $7 A 1$ is located downstream of the $5^{\prime}$ end of the adult $S \times l$ transcription unit (see below), we presume that the $S \times l$ gene may still be transcribed at some level in the $7 A 1$ mutant males and that some processing defect results in
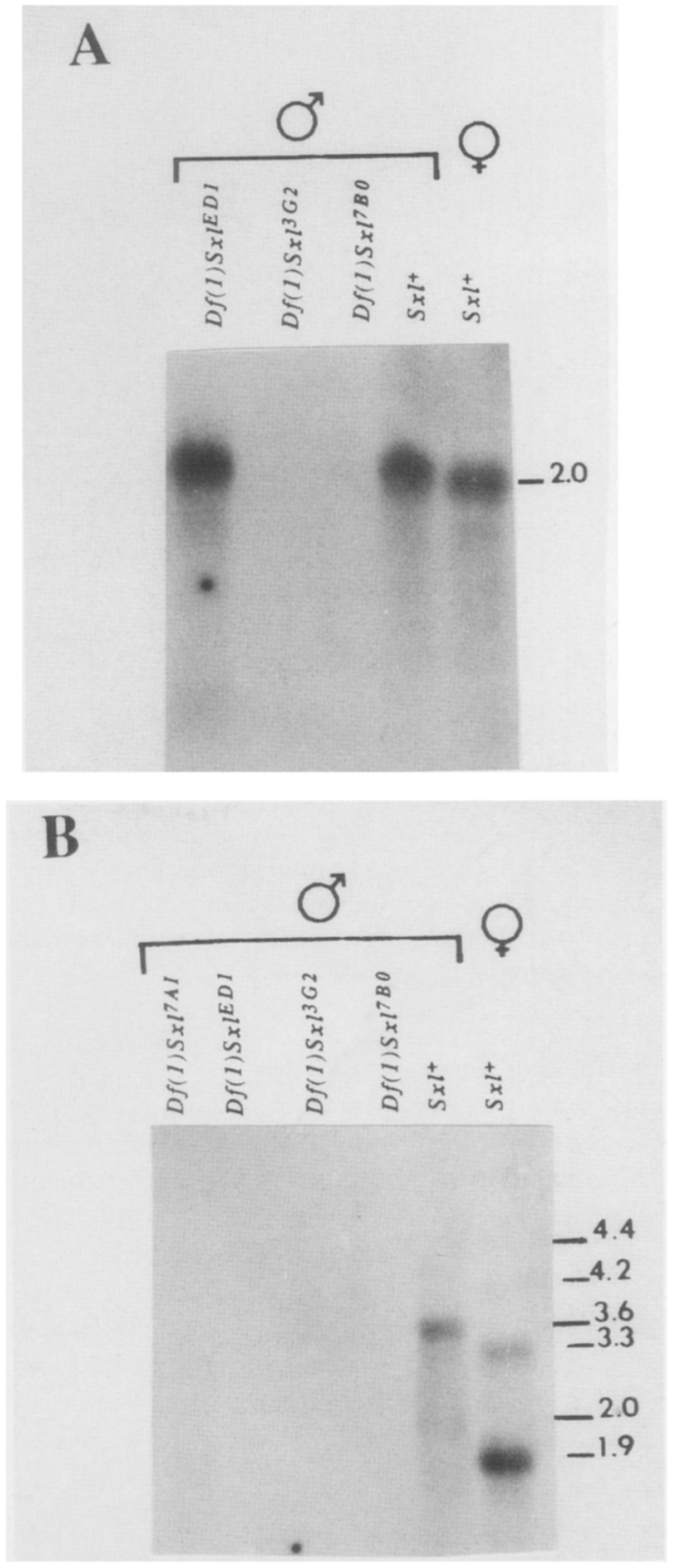

Figure 3. Expression of transcripts in males hemizygous for several $S x l$ deletion chromosomes (for a complete description of these deficiences, see Fig. 1 and Salz et al. 1987.) (A) The blot was hybridized with fragment $d$ (Fig. 1), which detects a 2-kb non-sex-specific RNA; $(B)$ the blot was hybridized with fragment h2 (Fig. 4), which detects several sex-specific RNAs. 


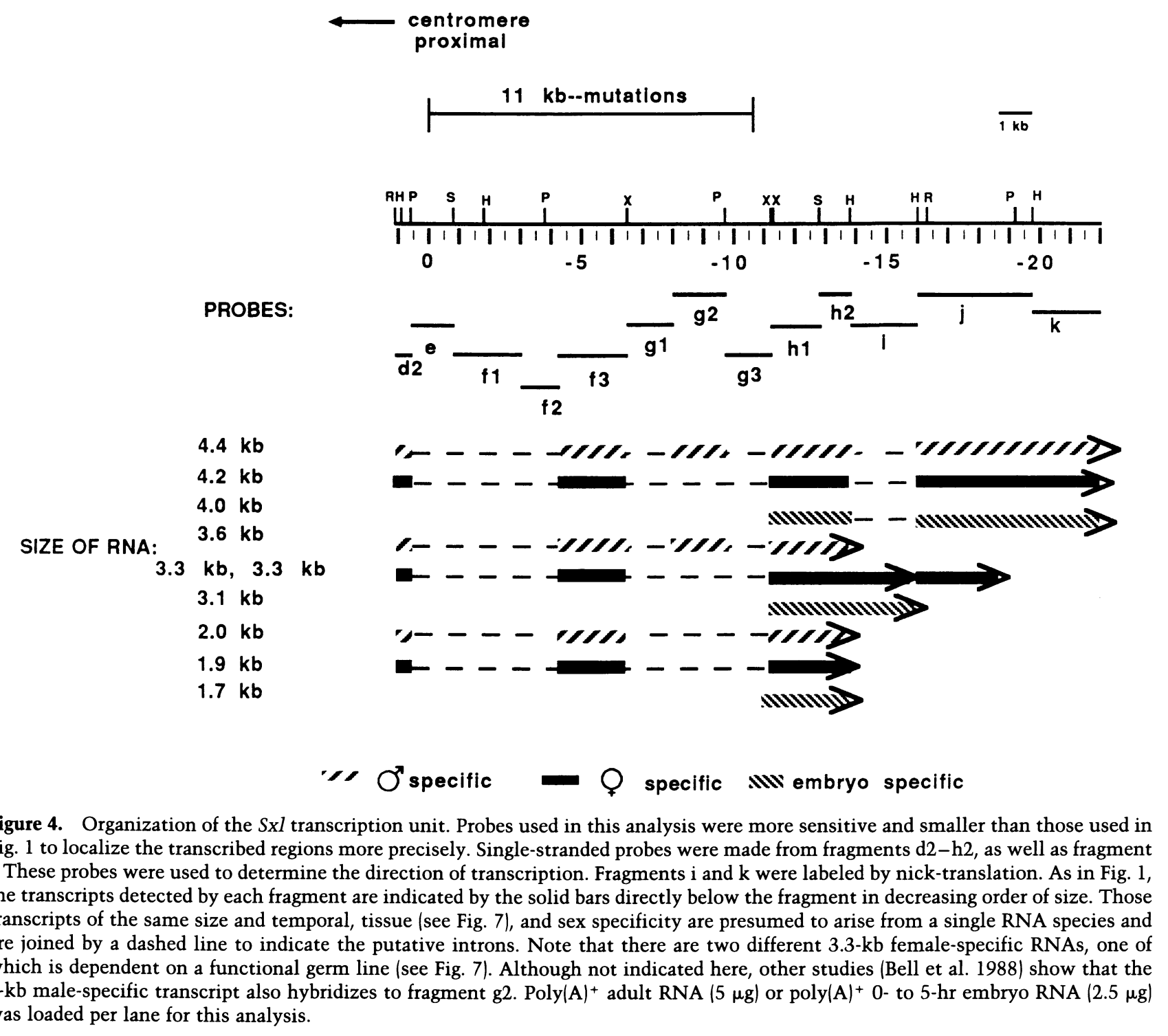

the production of abnormal RNAs.

Finally, in other experiments (data not shown), we have examined the expression of the distal 2.0- and 1.9$\mathrm{kb}$ RNAs labeled by fragment 1 . We found that both of these RNAs are expressed in males carrying deficiencies that delete large portions of the $S x l$ transcription unit (e.g., $7 B 0$ and 3G2) but retain fragment 1 DNA. Taken together, these findings indicate that the large transcription unit near the center of our chromosomal walk corresponds to the $S x l$ gene and that the RNAs encoded by the flanking proximal and distal regions must be encoded by transcription units distinct from Sxl.

\section{Organization of the Sxl transcription unit}

To define the $S x l$ transcription unit more precisely, smaller and more sensitive single-stranded probes were used to examine $S x l$ RNAs in embryos and adults. The results of this analysis are summarized in Figure 4. The salient findings are as follows: First, all of the $S x l$ RNAs are transcribed in the same direction, proximal to distal. Second, the Sxl transcription unit appears to extend over a DNA segment of at least $23 \mathrm{~kb}$. Third, the three size classes of RNA observed in adults clearly differ in structure. Most of the differences between the size classes appear to arise toward the $3^{\prime}$ end of the transcription unit in the region distal to fragment hl (see Fig. 4). For instance, fragment $\mathrm{i}$ hybridizes to the RNAs in the $3-\mathrm{kb}$ size class but does not hybridize to either the $\sim 2$ - or $\sim 4$-kb size classes. Similarly, only the largest size class $(\sim 4 \mathrm{~kb})$ hybridizes to fragment $\mathrm{k}$. Fourth, the male and female RNAs in each size class hybridize (with one important exception; see below) to the same set of restriction fragment probes. Hence, at least at the level of resolution provided by Northern analysis, it appears that there is a close correspondence in structure between the male- and female-specific RNAs in a given size class. As indicated in Figure 4, the structure of the embryonic RNAs also appears to be closely related to that of the adult transcripts. 
Two other results of the Northern analysis are of interest. The first is the probable location of the $5^{\prime}$ end of the $S x l$ transcription unit. As indicated in Figure 4 and illustrated in the Northern blot shown in Figure 5A, the 5 '-most probe that hybridizes to the adult Sxl RNAs is fragment $\mathrm{d} 2$. $\mathrm{d} 2$ is located proximal to coordinate 0 (see Fig. 4), and this would place the start site for the $S x I$ transcription unit upstream of both the P-element insertion of $S x f^{f P b}$ and the proximal breakpoint of $D f(1) S \times l^{7 A 1}$ (see Figs. 1 and 4). Although d2 labels all three size classes of Sxl RNA in both adult males and females, it does not hybridize to any of the embryonic-specific RNAs (see Fig. 5A). This raises the possibility that the embryonic transcripts may have a different $5^{\prime}$ start site from that of the adult RNAs. The existence of a distinct embryonic promoter was also suggested by genetic studies on Sxl deletion mutants (Salz et al. 1987).

The second result of interest is the difference between the adult male and female $S x l$ transcripts. Although the Northern experiments suggest that the male and female RNAs within a size class have similar structures, the male RNAs are $\sim 200 \mathrm{bp}$ larger than the corresponding female RNAs in each case (cf. Fig. 2). The difference between male and female RNAs appears to be due, at least in part, to the presence of an additional exon in the male RNAs. In the experiment shown in Figure 5B, RNA from adult males and females was probed with fragment $\mathrm{g} 2$, which is located near the middle of the $S x l$ transcription unit (see Fig. 4). This fragment does not hybridize to any of the female $S \times l$ transcripts, but it does label the 4.4and 3.6-kb Sxl male RNAs (see Fig. 5B). Though the 2.0$\mathrm{kb}$ male $\mathrm{Sxl}$ RNA is not observed in this Northern blot, it is much less abundant than the two larger male species (see Fig. 2). We suspect that the low level of the small transcript probably accounts for our inability to detect it with the genomic g2 probe. [In other experiments we have found that the $S \times 1$ exon in g2 labels all three male RNAs (Bell et al. 1988).]

\section{Developmental profile of the Sxl transcripts}

The Northern experiments described above indicate that the $S x l$ transcript pattern in 0- to 5-hr embryos differs from that in adults. It consists of two types of RNA: (1) a set of embryo-specific RNAs and (2) a set of RNAs which, by size and pattern of hybridization with different probes, appears to correspond to the $S x l$ transcripts found in adult females. We do not, however, detect transcripts corresponding to RNAs observed in adult males. These differences suggested that it would be of interest to learn more about the expression of the embryo RNAs and the transition to the transcript pattern found in adult females and males.

In the Northern blot shown in Figure 6, we have examined Sxl transcripts during the course of embryonic development. The transcript pattern in $0-$ to $2.5-\mathrm{hr}$ and $2.5-$ to $5-\mathrm{hr}$ embryos is roughly similar to that found in the longer 0- to 5-hr collections (cf. Fig. 6A with Fig. 2): It consists of a mixture of embryo-specific and adult female-specific transcripts. The $0-$ to $2.5-\mathrm{hr}$ and $2.5-$ to

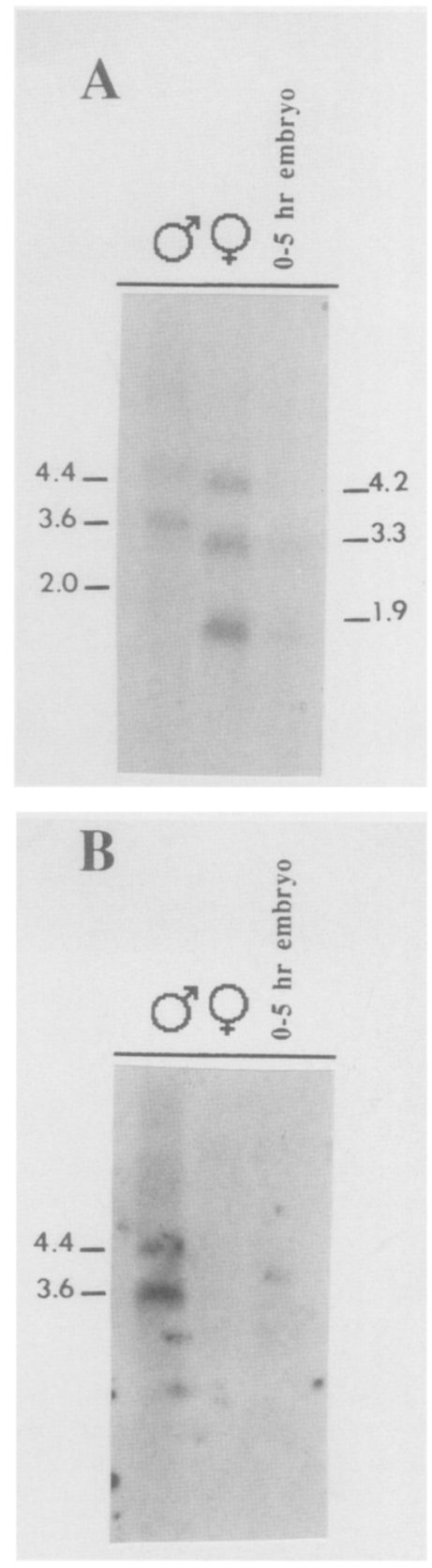

Figure 5. Identification of regions within the $S x l$ transcription unit that are specific to different classes of RNAs. $(A)$ The blot was hybridized with fragment $\mathrm{d} 2$ (see Fig. 4 ); $(B)$ the blot was hybridized with fragment g2 (see Fig. 4). In each case, $5 \mu \mathrm{g}$ poly $(\mathrm{A})^{+}$adult RNA or $2.5 \mu \mathrm{g}$ poly $(\mathrm{A})^{+}$embryo RNA was loaded per lane.

5-hr embryos do, however, show differences in the relative level of embryo-specific and female RNAs. The embryo-specific RNAs are more abundant in the 2.5- to 5-hr collection, whereas the female RNAs are more abundant in the earlier collection.

This observation suggested that it would be useful to determine the relative contributions of maternal and zygotic RNAs to the $S x l$ transcript pattern during the first 

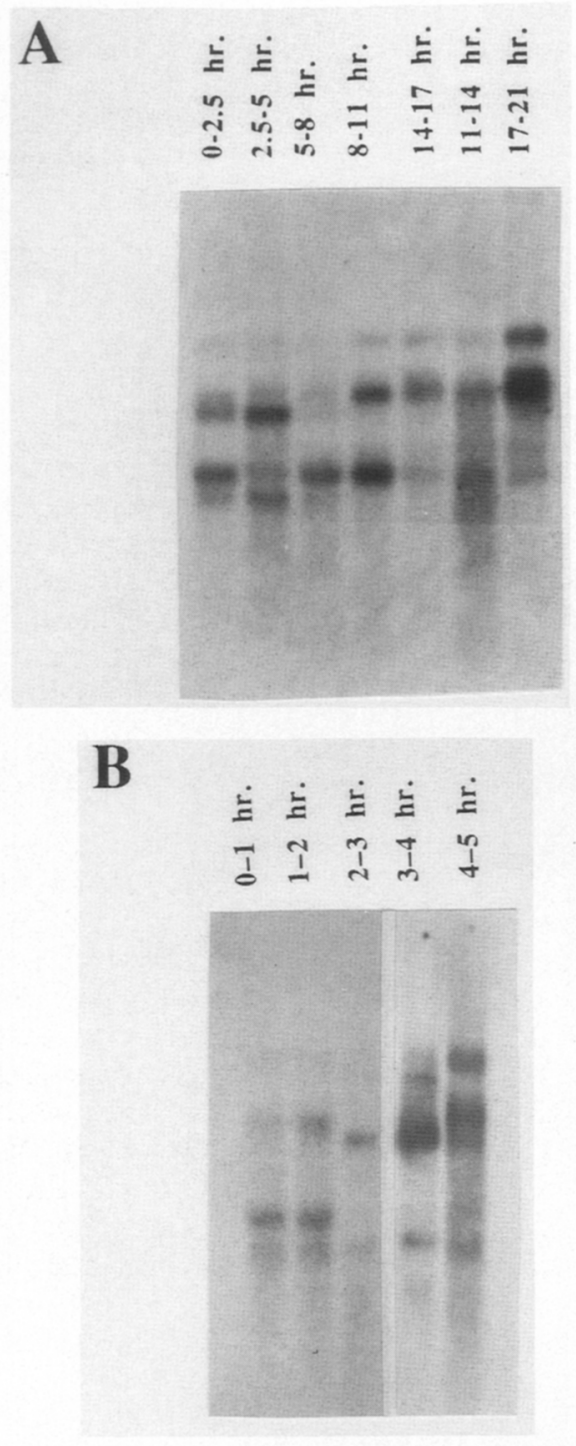

Figure 6. Developmental profile of $S_{x l}$ transcripts during the embryonic period. Fragment $h$ was used as a probe because it detects the majority of the $S x l$ transcripts (Fig. 1). All lanes contain $5 \mu \mathrm{g}$ poly $(\mathrm{A})^{+}$RNA made from staged embryos at $25^{\circ} \mathrm{C}$.

several hours of embryogenesis. As shown in Figure 7A, the female-specific RNAs in early embryos are found in unfertilized eggs and are likely to be of maternal origin. As can be seen in the detailed developmental profile shown in Figure 6B, these maternally derived female RNAs persist through the first $2 \mathrm{hr}$ of embryogenesis. During the next hour $(2-3 \mathrm{hr})$ the maternal female RNAs disappear and are replaced by the embryonic species. We presume that these embryonic transcripts are the first products of zygotic transcription of the $\mathrm{Sxl}$ gene. The level of the embryonic RNAs appears to peak in the 3- to 4-hr interval.

After this point, the early embryonic RNAs must turn over rather rapidly as these transcripts cannot be detected in 5- to 8-hr embryos (see Fig. 6A). Instead, we observe RNAs corresponding to the three size classes found in adult females. In the 5- to 8-hr collection, the most abundant $S \times 1$ transcript is the $1.9-\mathrm{kb}$ female-specific RNA, whereas the two larger species are only weakly labeled (cf. Fig. 6A and Fig. 2). In the 8- to ll-hr period, this pattern begins to change: The relative level of the two large female RNAs increases, and the level of the small RNA drops. In addition, transcripts corresponding in size to the adult male RNAs can first be detected during this time (e.g., the faint band just above the $3.3-\mathrm{kb}$ female RNA in the 8- to 11-hr lane). In the later stages of embryogenesis, the two larger female

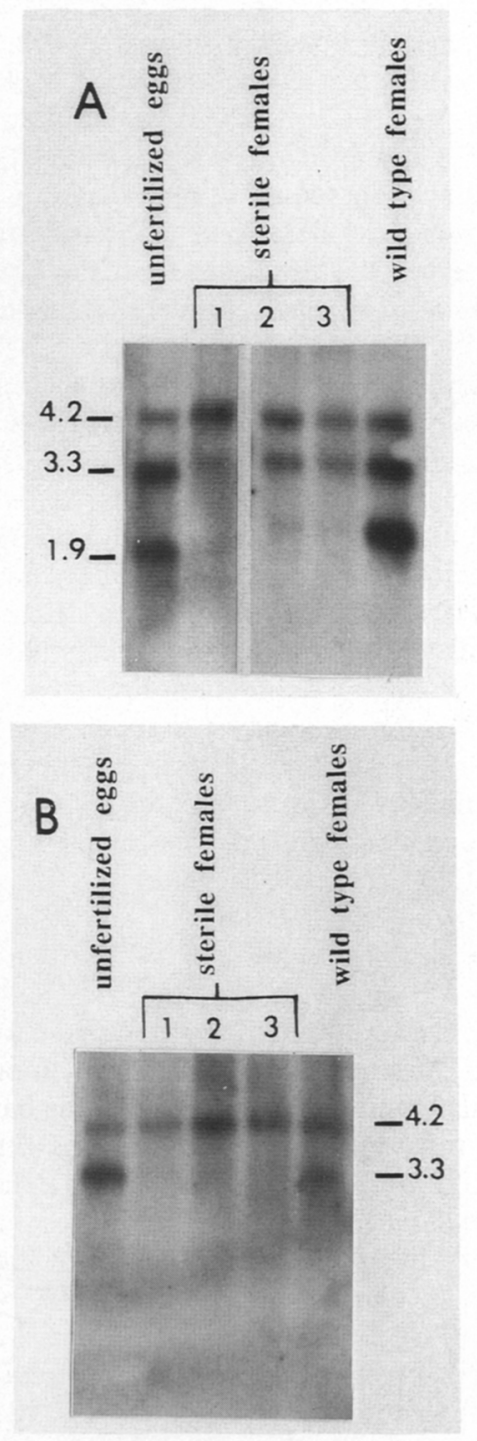

Figure 7. Sxl transcripts in unfertilized eggs and females without a functional germ line. Poly $(A)^{+}$unfertilized egg RNA $(2.5 \mu \mathrm{g})$ or poly $(\mathrm{A})^{+}$female RNA $(5 \mu \mathrm{g})$ were loaded per lane. The genotypes of the females without a functional germ line are as follows: (Lane 1) $\mathrm{Sxl}^{f^{s 1} 1 / \mathrm{Sx}^{f s 1}}$; (lane 2) $\mathrm{ovo}^{D 1 /}+$; (lane 3) females $\left(\right.$ tudor $\left.^{1} /+\right)$ from females homozygous for the maternal effect mutation tudor ${ }^{1}$. (A) The blot was hybridized with fragment $h_{;}(B)$ the blot was hybridized with fragment $j$ (Fig. 4). 
transcripts become the predominant $S x l$ RNAs, whereas the small female RNA becomes a minor species. The relative abundance of the two larger male Sxl RNAs also increases during this period.

We have also examined $S x l$ RNAs in first instar larvae, in sexed second and third instar larvae, and in pupae (Maine 1984). The transcript pattern in the larval and pupal stages is quite similar to that found in $17-$ to $21-\mathrm{hr}$ embryos: The major transcripts correspond to the larger $(3$ and $4 \mathrm{~kb}$ ) female and male size classes, whereas the small $(2 \mathrm{~kb})$ size class is barely detectable. Moreover, in the sexed second and third instar animals, we find that these transcripts are sex specific: Male larvae have transcripts that appear to be identical to those observed in adult males, whereas female larvae have transcripts that correspond to RNAs found in adult females.

\section{Germ-line dependence of Sxl transcripts}

Sxl is known to be required in the female germ line (Schüpbach 1985), and mutations that differentially affect this aspect of its function have been identified (Salz et al. 1987). Hence, it was of interest to compare the $S x l$ transcript pattern in wild-type females with that of females lacking a functional germ line. Sterile adult females that differ in the severity of their germ-line defect were generated in three different ways: (1) Females lacking germ cells altogether were collected as progeny of mothers homozygous for tudor ${ }^{1}$ (Boswell and Mahowald 1985); (2) females with abnormal germ cells were collected as $O V O^{D 1}$ heterozygotes (Busson et al. 1983; Oliver et al. 1987); (3) females with germ cell abnormalities because of defects in $S x l$ function were obtained as homozygotes for the viable but sterile partial loss-offunction $S x l$ allele, $S x I^{f s 1}$. [The abnormal egg chambers in the ovaries of $S x I^{7 s 1}$ females closely resemble those observed in chimeric females whose germ line is homozygous for a $S x l$ null mutation but whose soma is wild type for Sxl (Salz et al. 1987).]

Figure 7A shows the $S x l$ transcript pattern in wildtype and germ-line-deficient females detected with probe $h$. All of these sterile females exhibit abnormalities in their Sxl RNAs. First, the most abundant transcript in adult females, the 1.9-kb RNA, is barely detectable. In this respect, the transcript pattern in these mutant females closely resembles that found in the larval (and also pupal) stages when the female germ line is much less developed. Second, there is a reduction in the level of the $3.3-\mathrm{kb}$ size class relative to the $4.2-\mathrm{kb}$ size class. Further analysis has revealed that the reduced level of labeling of the $3.3-\mathrm{kb}$ band is due to the fact that this size class is composed of two different RNA species in wild-type females, one of which is absent in the sterile females. This is shown in the Northern blot probed with fragment $j$ (see Fig. 7B). In wild-type females and also in unfertilized eggs, probe $\mathrm{j}$ hybridizes to a 4.2and a $3.3-\mathrm{kb}$ band; in contrast, the $3.3-\mathrm{kb}$ band is missing in RNA from sterile females, whereas the $4.2-\mathrm{kb}$ band remains. We therefore conclude that there are four rather than three transcripts in adult females. The level of two of these is dependent on the presence of a functional germ line; however, all four transcripts appear to be present in unfertilized eggs.

\section{The sex-specific adult transcription pattern is not} affected by tra or tra-2

Sxl is upstream of both tra and tra-2 in the regulatory hierarchy. Thus, we would predict that mutations in either tra or tra-2 would not affect the Sxl transcription pattern. To test this hypothesis, we examined the pattern of transcription in XX animals that were sexually transformed by mutations in either tra or tra-2. We found that the $S x l$ transcript pattern was essentially female, in agreement with the genetic model (Fig. 8). The relative level of the 3.3- and the 1.9-kb RNAs compared to the 4.2-kb RNAs is similar to that seen in females without a functional germ line. This is not unexpected because these sexually transformed animals do not have a functional germ line.

\section{Discussion}

The studies reported here provide some of the first insights into the underlying molecular basis for the genetic complexity of $S x l$. We have identified the $S x l$ transcription unit and defined its probable limits. The transcription unit spans a DNA segment of at least $23 \mathrm{~kb}$ located in the $6 \mathrm{~F}$ cytogenetic interval of the $\mathrm{X}$ chromosome. Many of the DNA lesions associated with altered Sxl function map entirely within this 23-kb DNA segment, and we have shown that such lesions alter the $S \times l$ transcript pattern in a number of cases. The $S x l$ transcription unit is flanked on both the proximal and distal

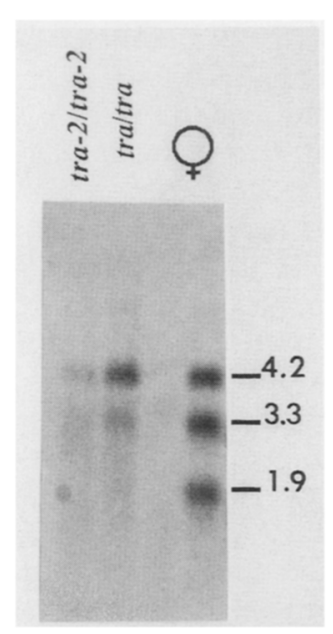

Figure 8. $S x l$ transcripts in females sexually transformed by the 'downstream genes' tra and tra-2. All lanes contain $5 \mu \mathrm{g}$ of poly $(\mathrm{A})^{+}$RNA. The blot was hybridized with a $S x l$ cDNA described in Bell et al. (1988). tra/tra sexually transformed females were isolated from the following cross: $y w f / y w f$; mwh tra/TM5 $\times+/ Y ; D f(3 L) s t^{81 k 17}($ tra- $) / T M 6 b$. tra-2/tra-2 sexually transformed females were isolated from the following cross at $29^{\circ} \mathrm{C}: \mathrm{y} / \mathrm{y}$; tra-2/SM1 $\times+/ B^{s} Y$; tra- $2^{\text {ts }} / \mathrm{CyO}$. 
sides by RNA-coding regions whose transcripts show no apparent sex and/or stage specificity. The RNAs produced by these flanking regions do not appear to be affected by mutations in $S x l$ that either eliminate or alter $S x l$ transcripts, and we conclude that they are encoded by genes that are distinct from $S x l$. The functions of the genes in the flanking regions are unknown. Although extensive efforts have been made to identify vital genes in the vicinity of $S x l$ (Nicklas and Cline 1983), none of the genes discovered in mutant screens would be sufficiently close to $S x l$ to correspond to any of the flanking transcription units. In addition, some of the deletions extending from $S x l$ into the flanking regions have no apparent effect on male viability or fertility under laboratory conditions.

We have found that the $S x l$ transcription unit encodes a complex set of overlapping RNA species that differ in their stage and/or sex specificity. Using Northern blots to analyze the transcript pattern during the Drosophila life cycle, we have been able to detect 10 different $S x l$ RNA species. This is probably an underestimate of the number of distinct $S x l$ transcripts, as there may be RNAs of similar size with subtle structural differences that were not detected by our Northern analysis.

Given the complexity of $S x l$ function that has been revealed by genetic analysis, it is intriguing that the gene encodes multiple transcripts showing sex, stage, and tissue specificity. Although it unclear at this point how the specific transcripts relate to the different $S x l$ functions, the transcriptional complexity provides some valuable clues about the regulation and functioning of Sxl during development.

\section{Male/female differences}

Even though $S x l$ must be on in females and off in males, the gene is transcribed in both sexes. In adult females there are four major RNA species: a large 4.2 -kb transcript, two different medium-sized transcripts of $3.3 \mathrm{~kb}$, and a small $1.9-\mathrm{kb}$ transcript. In adult males there are three slightly larger RNA species of $4.4,3.6$, and $2.0 \mathrm{~kb}$ transcribed off of the same strand as the female RNAs. At the level of resolution provided by Northern analysis, the large, medium, and small adult male transcripts appear very similar (but not identical) in structure to the large, to one of medium-sized, and to the small female transcript, respectively (a male transcript corresponding to medium-sized female germ-line-dependent RNA cannot be detected). The three male RNAs and the corresponding female RNAs are present at other stages in the Drosophila life cycle. In second and third instar larva, we have been able to establish the sex specificity of these transcripts. The male and female RNAs are also observed in mid- to late embryos and in pupae; we suspect that the sex specificity of the $S x l$ RNAs is maintained in these developmental stages as well but this has not yet been experimentally established.

Although the male and female $S x l$ RNAs appear to have quite similar structures, one consistent difference can be detected: Male RNAs hybridize to a restriction fragment probe derived from near the middle of the $S_{x l}$ transcription unit, whereas female RNAs do not. This would suggest that the male transcripts differ from the female RNAs by the presence of an additional internal exon. This suggestion has been confirmed in studies reported elsewhere (Bell et al. 1988). All three of the male RNAs contain a short $(\sim 180 \mathrm{bp})$ exon that is located immediately downstream from the exon containing the presumptive translation initiation signal for the $S \times 1$ proteins. Within the male exon are in-frame translation stop signals that interrupt the large open reading frame. Thus, the male RNAs would encode a truncated, and presumably nonfunctional, protein product. In contrast, this exon is spliced out of the female transcripts. This would conserve the large open reading frame and would, in principle, permit the synthesis of fully functional protein products. Because adult forms of the $S x l$ transcripts appear to be present from mid-embryogenesis to the adult stage, the on/off regulation of $S x l$ during most of the Drosophila life cycle is apparently achieved by the alternate splicing of the male exon. Moreover, $S x 1$ itself most probably participates in the splicing process. A feature common to the proteins encoded by the various female $S x l$ transcripts is two domains showing extensive sequence similarities to a family of RNA-binding proteins (Bell et al. 1988). This observation would suggest that the positive autoregulatory function of $S x l$ may involve some type of interaction of the $S x l$ protein products with the splicing machinery to confer the female-specific splicing pattern on the late $S x l$ transcripts. In contrast, when the $S x l$ products are absent, the $S x l$ transcripts would be spliced in the male pattern (Bell et al. 1988).

\section{Germ-line/soma differences in adult females}

Two of the four $S x 1$ RNAs found in adult females show a striking dependence on the presence of a functional germ line. In the absence of a germ line, the small 1.9-kb RNA is present at much lower levels and one of the medium sized 3.3-kb RNA species cannot be detected at all. The simplest interpretation of these observations is that these two RNAs are expressed primarily in the germ line or ovary of adult females, whereas they are expressed either at only very low levels or not at all in the rest of the animal. Consistent with this notion, we find that both of these RNAs are present in unfertilized eggs. The other two adult female transcripts (the $4.2-\mathrm{kb}$ and other medium sized 3.3-kb RNA) are also present in unfertilized eggs. Hence, it seems likely that the latter two transcripts are expressed in both germ line and soma.

The germ-line dependence of two of the female transcripts is intriguing in light of the fact that $S \times l$ is required for germ-line development and function. Genetic studies suggest that the role of $S x l$ in the germ line may be complex (Salz et al. 1987). Several alleles of $S x l$ have been identified that appear to be specifically defective in germ-line function, but wild type with respect to somatic function. One of these $S x 1$ alleles, $S x l^{f s 1}$, has been characterized in detail (Salz et al. 1987 and H. Salz, un- 
publ.). Females homozygous for this viable but sterile allele produce abnormal egg chambers that are similar in phenotype to the cystic egg chambers observed in germ cells homozygous for a Sxl null allele in a wild-type somatic environment (Schüpbach 1985). Surprisingly, the female sterility of $S x l^{s i 1}$ is complemented by Sxl deletions that eliminate portions of the adult transcription unit. Three deletions that remove sequences proximal to coordinate 0 (see Fig. 1), and thus lack the probable $5^{\prime}$ end of the $S \times 1$ transcription unit (in fragment d2 of Fig. 4.), complement the female sterility of this allele (Salz et al. 1987). Because the female sterility of $S x l^{f s 1}$ is also rescued by small internal deletions extending from coordinate 0 up to -6.5 , it would appear that sequences sufficient for complementation are located $3^{\prime}$ to coordinate -6.5 (Salz et al. 1987). One hypothesis suggested by these findings is that $S \times l$ has an internal promoter and/ or transcription start site that can be used in the germ line. Clearly, further studies are required to determine whether this is the molecular basis for the observed complementation.

Although the deletion mutants complement the female sterile $S x l^{\text {ss } 1}$ allele, they lack some essential aspect of $S x l$ activity in the germ line, as they are unable to support normal germ-line development in homozygous clones (Salz et al. 1987). We have found that these same deletions eliminate the three $S x l$ transcripts in adult males. Presumably, the corresponding female transcripts would be absent in homozygous mutant female germline clones. Hence, at least one of the adult female RNAs is likely to be involved in this aspect of Sxl's germ-line function.

\section{Maternal/zygotic transition}

Although $S x l$ functions in both the maternal germ line and the early embryo, genetic experiments have not revealed any clear-cut $S \times 1$ maternal effect that would suggest a role for maternal $S x l$ products in zygotic sex determination (see Cline 1980; Oliver et al. 1988). Thus, we had not anticipated finding the maternally derived adult female $S x l$ transcripts in unfertilized eggs and very early embryos. Given the potentially deleterious effects that functional $S x l$ products would have on chromosomally male embryos as a consequence of $S x l$ autoregulation (Cline 1984), we presume that these maternally derived Sxl transcripts (and/or their protein products) are either nonfunctional in the embryo or are inactivated prior to the time when the adult or late transcripts are first expressed in the embryo and the commitment to the male or female developmental pathway is firmly established. It is, however, conceivable that the maternally derived $S x l$ products might be required at a point earlier in embryogenesis for the initial response of the zygotic $\mathrm{Sxl}$ gene to the $\mathrm{X} / \mathrm{A}$ signal (see below). In this respect, it is of interest that the maternally derived $S x l$ RNAs persist only during the first $2 \mathrm{hr}$ of development, disappearing at or just prior to the time when the X/A signal is first assessed and the activity state of $S x l$ is selected initially. It will be important to determine whether the translation products of these maternal $S x l$ RNAs are also found for a brief period during very early embryogenesis.

\section{Early/late transcription differences}

The functioning of Sxl very early in development when its activity state is first selected in response to the $\mathrm{X} / \mathrm{A}$ signal is genetically separable from its functioning during the remainder of development. During the first few hours after fertilization in chromosomal females, $S x l$ interacts with $d a$, sis-a, and sis-b, as well as with itself, and there are mutations in $S \times l$ that differentially affect these early interactions (Cline 1976, 1984, 1986, 1988a; Maine et al. 1985b; Salz et al. 1987). Moreover, there is an early phase of X chromosome dosage compensation controlled by $S \times l$ that is distinct from later phases (Gergen 1987).

In view of these special requirements for $\mathrm{Sxl}$ activity during the early phase of embryogenesis, it is particularly intriguing that there is a distinct set of embryonic or early Sxl transcripts. Because these early RNAs appear to be the first products of zygotic $S x l$ transcription, we suppose that they provide the early $S x l$ functions that have been identified in genetic experiments. Our experiments show that the early RNAs can first be detected after $\sim 2 \mathrm{hr}$ postfertilization. They are present for approximately a 2 -hr period corresponding to the interval in which the early $S x l$ functions are likely to be required. At $\sim 4 \mathrm{hr}$ after fertilization, there is a transition to the late phase of $S x l$ expression: The embryonic RNAs disappear, and we begin to detect the late, or adult, female-specific RNAs.

Although our results suggest that the early RNAs share the same core region as the late transcripts /see Fig. 4), we note two potentially important differences. First, the $5^{\prime}$-most exon of the late RNAs appears to be located in fragment $\mathrm{d} 2(+1$ to +0.5$)$. This exon does not appear to be present in the early transcripts, suggesting that they initiate at a site different from that used by the late transcripts. This possibility is supported by the analysis of $S \times l$ deletion mutants generated in a P-element mobilization scheme (Salz et al. 1987). Deficiencies that remove sequences proximal to the P-element insertion site at coordinate 0 , including the probable $5^{\prime}$ adult exon, are defective in late somatic Sxl functions but fully complement $S \times I^{f 9}$, an allele specifically defective in early Sxl functions (Maine et al. 1985b; Cline 1986). These results would argue that the transcription start site for the early RNAs is internal, somewhere downstream of the probable adult start site in $\mathrm{d} 2$. Consistent with this notion, when we selected for $S x l$ mutants that failed to complement $S \times f^{f \rho}$, we recovered several internal deficiencies that remove sequences extending from coordinate 0 to a DNA segment around coordinate -6 . One plausible interpretation of this observation is that there is an essential promoter/enhancer element or the transcription start site for the early RNAs located in the DNA segment from coordinate 0 to -6 .

A second difference is that none of the early RNAs appears to contain the male-specific exon (which is located in fragment $\mathrm{g} 2$ between coordinates -8 and -10 : see Fig. 4). A possible explanation for this finding is that the early transcripts might initiate at a site downstream of the male-specific exon. However, preliminary analysis of partial embryonic cDNAs (L. Keyes, unpubl.) sug- 
gests that is not the case and that the early transcripts start at a site located upstream of the male-specific exon, somewhere in the region between coordinates 0 and -6 . If this is correct, the male exon must be spliced out of the early transcripts. This would raise important questions about the possible mechanism for such a splicing process: Is it a structural feature of the early transcripts that permits the splicing machinery to skip over the male exon in the absence of $S x l$ products, or are some residual maternally derived $S x l$ proteins involved in the splicing of the early transcripts? It may be possible to answer these questions once we have a better understanding of the structure of the early RNAs.

The findings reported here raise the possibility that $S x 1$ regulation early in development may be quite different from the alternate splicing mechanism used during most of the life cycle. Thus, the initial response of $S \mathrm{x} l$ to the X/A signal may be at the level of transcription, resulting in the expression of the early $S_{x} 1$ RNAs in female but not in male embryos. Although conclusive evidence in support of sex-specific early transcriptional regulation will require probes specific for the embryonic RNAs, it should be noted that in situ hybridization experiments with a probe that labels all $S x l$ RNAs indicate that there are two classes of blastodermstage embryos: those that show strong hybridization and those that show weak hybridization (D. Coulter, unpubl.). What would the function of the early $S x l$ products be, and what mechanisms would account for the establishment of a stable commitment to either the female or male developmental pathway? Presumably, the proteins produced from the early transcripts would be responsible for the early dosage-compensation functions of $S x l$ and would facilitate the stable activation of $S x l$ in females. For example, when $S x l$ transcription switches from the early to the late phase, $\sim 4 \mathrm{hr}$ after fertilization, the early $S x l$ proteins might be involved in promoting the femalespecific splicing pattern of the newly synthesized late transcripts. Once the late $S x l$ proteins are processed, the autoregulatory functions of $S x l$ would then maintain a stable commitment to the female developmental pathway. In contrast, in male embryos, no functional early $S x l$ proteins would be present during the transition to the late phase, and the late transcripts would be spliced in the male mode with the male-specific exon included. It remains to be determined whether the somewhat delayed appearance of the late male transcripts in embryos reflects some type of transient sexspecific transcriptional regulation of the late promoter or arises from a decreased stability of RNAs containing a truncated open reading frame. Further studies will be required to test our model for the regulation of $S x l$ and also to determine the functional role of the different $S x l$ transcripts.

\section{Materials and methods}

Fly stocks

All crosses were carried out at $25^{\circ} \mathrm{C}$ on a standard cornmeal, dextrose, yeast medium (Cline 1978). Genes not listed in Lindsley and Grell (1968) are referenced in the text.
Recombinant DNA library construction, screening, and subcloning

The screening of recombinant phage libraries (to extend the walk described in Maine et al. 1985a) and restriction digests were done as described in Maine et al. (1985a). Subcloning of genomic fragments was done essentially as described in Maniatis et al. (1982).

\section{Genomic DNA isolation and Southern blotting}

DNA was isolated as described in Ish-Horowicz et al. (1979). Genomic blots were done as described by Maine et al. (1985a).

\section{RNA isolation and Northern blotting}

Poly $(\mathrm{A})^{+}$RNA was prepared by the method of O'Hare et al. (1983). In general, we used $5 \mu \mathrm{g}$ of poly(A) ${ }^{+}$RNA per lane. RNA was fractionated on denaturing $0.7 \%$ agarose $/ 6.7 \%$ formaldehyde gels made with a MOPS buffer, as described by Maniatis et al. (1982). RNA was transferred to nitrocellulose using $10 \times$ SSPE and hybridized using the same conditions as genomic Southern blots. Probes labeled with ${ }^{32} \mathrm{P}$ were made either by nick translation, random primer extensions of isolated fragments, or single-stranded DNA probes from M13 subclones. Single-stranded probes of $1 \mathrm{~kb}$ or less were made by extending from the M13 sequencing primer with Klenow. Single-stranded probes longer than $1 \mathrm{~kb}$ were extended using the modified $\mathrm{T} 7$ polymerase sequenase from USB (Cleveland, Ohio). The probe was cut with the appropriate restriction enzyme, denatured, isolated on a $1.0-1.2 \%$ low-melt agarose gel, melted, and added directly to the hybridization mix. The Sxl RNAs were sized (1) by reprobing with probes specific for the $5 \mathrm{C}$ actin gene $(1.95 \mathrm{~kb}$; Fyrberg et al. 1983), dorsal gene (2.8 kb; Steward et al. 1984), and rudimentary gene (7.3 kb; Segraves et al. 1983), and (2) by running the RNA alongside denatured DNA size standards in the range of $1-4 \mathrm{~kb}$.

\section{Acknowledgments}

We thank Drs. D. Coulter and L. Bell for stimulating discussions and sharing unpublished results. We are especially grateful to Dr. C. Cronmiller for donating several Northern blots for us to reprobe and for many inspiring discussions. We also thank Drs. M. Wolfner and T. Schüpbach for providing some of the stocks in this study. This work was supported by grants from the National Institutes of Health, the March of Dimes, and the American Cancer Society. M.E.S. is a Burroughs Welcome Fund fellow of the Life Sciences Research Foundation.

\section{References}

Baker, B.S. and J.M. Belote. 1983. Sex determination and dosage compensation in Drosophila melanogaster. Annu. Rev. Genet. 17: 345-393.

Baker, B.S. and K.A. Ridge. 1980. Sex and the single cell. On the action of major loci affecting sex determination in Drosophila melanogaster. Genetics 94: 383-423.

Bell, L.R., E.M. Maine, P. Schedl, and T.W. Cline. 1988. Sexlethal, a Drosophila sex determination switch, exhibits sexspecific RNA splicing and sequence similarity to RNA binding proteins. Cell 55: 1037-1046.

Boggs, R.T., G.P. Gregor, S. Idriss, J.M. Belote, and $M$. McKeown. 1987. Regulation of sexual differentiation in $D$. melanogaster via alternative splicing of RNA from the transformer gene. Cell 50: 739-747. 
Boswell, R.E. and A.P. Mahowald. 1985. tudor, a gene required for the proper assembly of the germ plasm of Drosophila melanogaster. Cell 42: 97-104.

Busson, B., M. Gans, K. Komitopoulou, and M. Masson. 1983. Genetic analysis of three dominant female sterile mutations located on the X-chromosome of Drosophila melanogaster. Genetics 105: 309-325.

Cline, T.W. 1976. A sex-specific, temperature-sensitive maternal effect of the daughterless mutation of Drosophila melanogaster. Genetics 84: 723-742.

- 1978. Two closely linked mutations in Drosophila melanogaster that are lethal to opposite sexes and interact with daughterless. Genetics 90: 683-698.

- 1979a. A product of the maternally influenced Sexlethal gene determines sex in Drosophila melanogaster. Genetics (suppl.) 91: s22 (Abstr.).

- 1979b. A male-specific mutation in Drosophila melanogaster that transforms sex. Dev. Biol. 72: 266-275.

- 1980. Maternal and zygotic sex-specific gene interactions in Drosophila melanogaster. Genetics 96: 903-926.

- 1983. The interaction between daughterless and Sexlethal in triploids: A lethal sex transforming maternal effect linking sex determination and dosage compensation in Drosophila. Dev. Biol. 95: 260-274.

- 1984. Autoregulatory functioning of a Drosophila gene product that establishes and maintains the sexually determined state. Genetics 107: 231-277.

- 1985. Primary events in the determination of sex in Drosophila melanogaster. In Origin and evolution of sex (ed. H.O. Halvorson and A. Monroy), pp. 301-327. Alan R. Liss, New York.

- 1986. A female-specific lesion in an X-linked positive regulator of the Drosophila sex determination gene, Sexlethal. Genetics 113: 641-663 (corrigendum 114: 345 ).

- 1988a. Evidence that sisterless- $a$ and sisterless- $b$ are two of several discrete 'numerator elements' of the X/A sex determination signal in Drosophila that switch $\mathrm{Sxl}$ between two alternative stable expression states. Genetics 119: 829862.

- 1988b. Exploring the role of the gene, Sex-lethal, in the genetic programing of Drosophila sexual dimorphism. In Evolutionary mechanisms in sex determination (CRC Uniscience Series) (ed. S.S. Wachtel), pp. 23-36. CRC Press, Boca Raton, Florida.

Fyrberg, E.A., J.W. Mahaffey, B.J. Bond, and N. Davidson. 1983. Transcripts of the six Drosophila actin genes accumulate in a stage- and tissue-specific manner. Cell 33: 115-123.

Gergen, J.P. 1987. Dosage compensation in Drosophila: Evidence that daughterless and Sex-lethal control X chromosome activity at the blastoderm stage of embryogenesis. $\mathrm{Ge}$ netics 117: 477-485.

Ish-Horowicz, D., S.M., Pinchin, P. Schedl, S. Artavanis-Tsakonas, and M.-E. Mirault. 1979. Genetic and molecular analysis of the $87 \mathrm{~A} 7$ and $87 \mathrm{Cl}$ heat-inducible loci of $D$. melanogaster. Cell 18: 1351-1358.

Lindsley, D. and E. Grell. 1968. Genetic variations of Drosophila melanogaster. Carnegie Inst. Wash. Publ. 627.

Lucchesi, J.C. and J.E. Manning. 1987. Gene dosage compensation in Drosophila melanogaster. Adv. Genet. 24: 371-429.

Lucchesi, J.C. and T. Skripsky. 1981. The link between dosage compensation and sex differentiation in Drosophila melanogaster. Chromosoma 82: 217-227.

Maine, E.M. 1984. 'A molecular analysis of the Sex-lethal locus in Drosophila melanogaster.' Ph.D. thesis, Princeton University, Princeton, New Jersey.

Maine, E.M., H.K. Salz, T.W. Cline, and P. Schedl. 1985a. The
Sex-lethal gene of Drosophila: DNA alterations associated with sex-specific lethal mutations. Cell 43: 521-529.

Maine, E.M., H.K. Salz, P. Schedl, and T.W. Cline. 1985b. Sexlethal, a link between sex determination and sexual differentiation in Drosophila melanogaster. Cold Spring Harbor Symp. Quant. Biol. 50: 595-604.

Maniatis, T., E.F. Fritsch, and J. Sambrook. 1982. Molecular cloning: A laboratory manual. Cold Spring Harbor Laboratory, Cold Spring Harbor, New York.

Meyerowitz, E.M. and D.S. Hogness. 1982. Molecular organization of a Drosophila puff site that responds to ecdysone. Cell 28: $165-176$.

Nagoshi, R.N., M. McKeown, K.C. Burtis, J.M. Belote, and B.S. Baker. 1988. The control of alternative splicing at genes regulating sexual differentiation in D. melanogaster. Cell 53: $229-236$.

Nicklas, J.A. and T.W. Cline. 1983. Vital genes that flank Sexlethal, an X-linked sex determining gene of Drosophila melanogaster. Genetics 103: 617-631.

Nöthiger, R. and M. Steinmann-Zwicky. 1985. Sex determination in Drosophila. Trends Genet. 1: 209-215.

O'Hare, K., R. Levis, and G. Rubin. 1983. Transcription of the white locus in Drosophila melanogaster. Proc. Natl. Acad. Sci. 80: 6917-6921.

Oliver, B., N. Perrimon, and A.P. Mahowald. 1987. The ovo locus is required for sex-specific germ line maintenance in Drosophila. Genes Dev. 1: 913-923.

-1988. Genetic evidence that the sans-fille locus is involved in Drosophila sex determination. Genetics 120: $159-171$.

Salz, H.K., T.W. Cline, and P. Schedl. 1987. Functional changes associated with structural alteration induced by mobilization of a P element inserted in the Sex-lethal gene of Drosophila. Genetics 117: 221-231.

Sanchez, L. and R. Nöthiger. 1982. Clonal analysis of Sexlethal, a gene needed for female sexual development in Drosophila melanogaster. Wilhelm Roux's Arch. Dev. Biol. 191: $211-214$.

-1983. Sex determination and dosage compensation in Drosophila melanogaster: Production of male clones in $X X$ females. EMBO I. 2: 485-491.

Scherer, G., C. Tschudi, J. Pierara, H. Delius, and V. Pirrotta. 1982. B104, a newly dispersed gene family in Drosophila melanogaster and its analogies with retroviruses. J. Mol. Biol. 157: 435-451.

Schüpbach, T. 1985. Normal female germ cell differentiation requires the female $\mathrm{X}$ chromosome to autosome ratio and expression of Sex-lethal in Drosophila melanogaster. Genetics 109: 529-548.

Segraves, W.A., C. Louis, P. Schedl, and B.P. Jarry. 1983. Isolation of the rudimentary locus of Drosophila melanogaster. Mol. Gen. Genet. 189: 34-40.

Steward, R., F.J. McNally, and P. Schedl. 1984. Isolation of the dorsal locus of Drosophila. Nature 311: 262-265. 


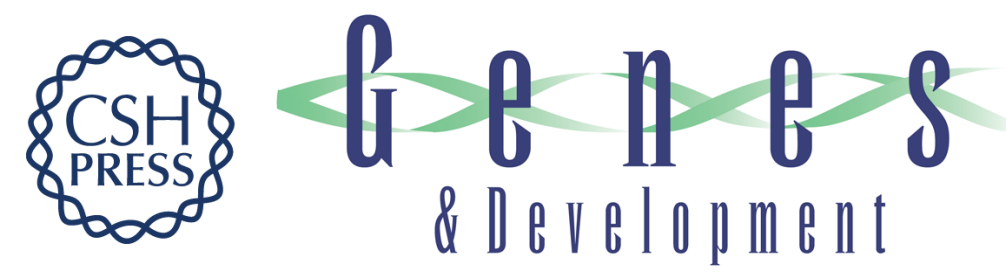

\section{The Drosophila female-specific sex-determination gene, Sex-lethal, has stage-, tissue-, and sex-specific RNAs suggesting multiple modes of regulation.}

H K Salz, E M Maine, L N Keyes, et al.

Genes Dev. 1989, 3:

Access the most recent version at doi:10.1101/gad.3.5.708

References This article cites 36 articles, 16 of which can be accessed free at:

http://genesdev.cshlp.org/content/3/5/708.full.html\#ref-list-1

License

Email Alerting Receive free email alerts when new articles cite this article - sign up in the box at the top

Service right corner of the article or click here.

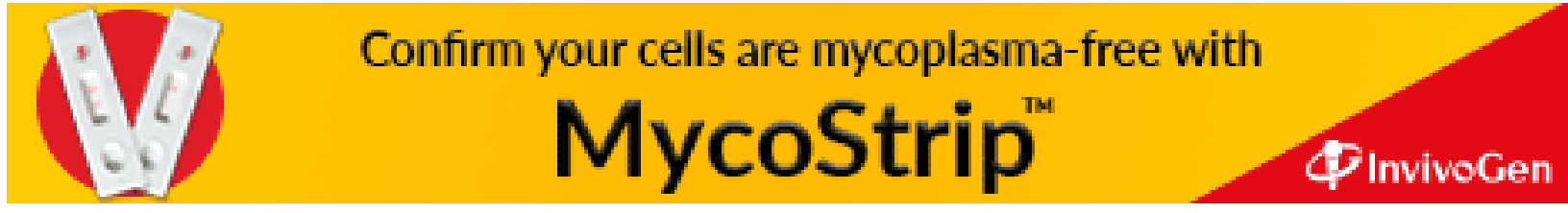

